\title{
TECHNO, UNE HISTOIRE DE CORPS ET DE MACHINES
}

Renaud Epstein, Jean Gaudillière, Irène Jami, Patricia Osganian, François Ribac

\section{La Découverte | «Mouvements »}

$2005 / 5 n^{0} 42$ | pages 5 à 8

ISSN 1291-6412

ISBN 2707147567

Article disponible en ligne à l'adresse :

http://www.cairn.info/revue-mouvements-2005-5-page-5.htm

\section{!Pour citer cet article :}

Renaud Epstein et al., «Techno, une histoire de corps et de machines », Mouvements 2005/5 ( ${ }^{\circ}$ 42), p. 5-8.

DOI 10.3917/mouv.042.0005

Distribution électronique Cairn.info pour La Découverte.

(C) La Découverte. Tous droits réservés pour tous pays.

La reproduction ou représentation de cet article, notamment par photocopie, n'est autorisée que dans les limites des conditions générales d'utilisation du site ou, le cas échéant, des conditions générales de la licence souscrite par votre établissement. Toute autre reproduction ou représentation, en tout ou partie, sous quelque forme et de quelque manière que ce soit, est interdite sauf accord préalable et écrit de l'éditeur, en dehors des cas prévus par la législation en vigueur en France. Il est précisé que son stockage dans une base de données est également interdit. 


\section{DOSSIER}

\section{Techno, \\ une histoire de corps et de machines}

I y a environ vingt ans, la techno a fait irruption dans la galaxie des musiques populaires. Suivant de près l'expansion du hip hop, cette nouvelle musique électronique s'est imposée comme l'une des composantes essentielles du paysage musical mondial, aux côtés du rock et du rap, donnant lieu à de nombreuses déclinaisons régionales et à un foisonnement stylistique considérable. A l'instar de toute culture musicale, la techno ne se limite pas à des sons. Elle recouvre tout un monde d'objets, d'outils, de lieux, de circulations, de façons d'être, de généalogies, de représentations, de savoirs. C'est cet assemblage qui en fait, plutôt qu'un style, un genre.

Cette nouvelle musique naît au milieu des années 1980 à Detroit (USA), capitale déclinante de l'industrie automobile, où des $D J s$ redécouvrent des répertoires des années 1970 (par exemple les groupes allemands Kraftwerk et Ash Ra Tempel) et utilisent divers instruments électroniques (ordinateurs personnels Atari, boîtes à rythme, synthétiseurs, magnétophones portables) pour expérimenter de nouveaux mélanges sonores. L'éclosion de la techno suivra rapidement, en passant de I'espace domestique aux dance-floors. Là, les DJs y rodent leurs nouveaux instruments, reprenant à leur compte certaines des techniques du hip hop comme le mixage des disques vinyles, et jettent les bases du nouveau genre. II en est de même pour les danseurs (euses) qui, au contact des pulsations électroniques, s'immergent plus longuement dans la musique et inventent un nouveau corps dansant, sans doute moins sexué qu'il ne l'était dans les night-clubs du disco. Détails d'importance, le mouvement naissant ne goûte guère les images, privilégie l'anonymat et, se méfiant de l'industrie musicale, s'appuie sur de nombreux microlabels qui se chargent de diffuser les nouvelles sonorités. 
La suite de l'histoire, et notamment celle du mouvement techno européen, est plus connue. Chassée des clubs et jalouse de son indépendance, la mouvance techno investit de manière inédite l'espace urbain. Utilisant à bon escient les réseaux de communication pour prévenir les amateurs, les organisateurs de raves $s^{\prime}$ approprient des friches industrielles, des terrains oubliés dans les interstices des villes ou des lieux retirés dans leurs marges. Le temps d'une nuit ou de quelques jours, des milliers de personnes se rassemblent dans ces lieux détournés de leur fonction initiale, pour les transformer en espaces de fêtes libres et gratuites (free parties). Lesquelles seront rapidement présentées comme des lieux de dépravation pour la jeunesse par les médias et les gouvernements. La dénonciation du cocktail sexe, drogue et musique à elle aussi une longue histoire.

La techno bénéficie désormais d'une attention soutenue de la part des journalistes et des « savants ». À la dénonciation des nuisances des fêtes techno et des risques qu'elles font peser sur leurs participants répond la célébration, par certains philosophes " post-modernes", de la plus grande révolution artistique du XXle siècle et de la crise de «l'œuvre ». Soit deux registres opposés mais qui partagent un point de vue surplombant sur la techno considérée comme un tout.

Autant le dire, nos préventions sont fortes à l'égard de cette approche qui considère la techno de l'extérieur. D'abord, parce qu'il est abstrait de parler d'une culture globalement. La techno n'a pas d'essence; autant que sa définition, ses formes varient sans cesse. Si la techno est devenue si influente, c'est justement parce qu'elle propose de multiples entrées aux amateurs; par le biais des disques et des répertoires, des fêtes, sous l'angle de l'internet, de la vidéo, du Dijaying, etc.

Ensuite, car n'en déplaise aux « post-modernes », la techno ne sort pas de nulle part. Surgie au moment de l'avènement du home studio et de l'essor de l'ordinateur personnel, la techno s'est d'emblée posée comme un art de la mémoire, faisant des supports enregistrés et des disques durs ses plus sûrs alliés. Captivé par les raves, l'observateur oublie trop souvent que les flux technoïdes passent aussi par les disques et l'internet. Justement, si nous nous approchons du dance floor, au cœur de la fête, que voyons nous? Un bien curieux mélange: des écrans et des lumières qui flashent, les corps de ceux et celles qui dansent, le DJ qui mixe dans une semi-obscurité, I'ordinateur qui contient tous les fichiers de toutes les musiques récupérées, copiées ainsi que les piles de disques vinyles, les colonnes d'enceintes empilées d'où sortent les pulsations, le stand de Médecins du Monde où l'on teste les « produits». Si maintenant, nous regardons par dessus la tête de l'amateur de drum'n bass en train de bidouiller à la maison, que distinguons nous? À peu près les mêmes objets mais en plus petits: l'ordinateur et la connexion à internet activés, des haut-parleurs disposés sur une table, un peu plus loin une platine disque et une pile de disques vinyles classés par labels...

Autrement dit, si nous désirons comprendre ce drôle d'édifice que l'on appelle techno, il faut le faire de l'intérieur. Cela suppose de s'intéresser aux raves, au club- 
bing, aux drogues, au peer to peer, aux labels, aux CD, aux samplers ainsi qu'aux corps, à leurs mises en scène et leurs mises l'épreuve. Mais il est aussi essentiel de ne pas oublier la sphère domestique (ce n'est pas pour rien que l'on parle de house music) et surtout se coltiner ces objets « techniques » que les sociologues omettent souvent. II y a tout lieu de penser que si nous négligeons ces pièces du dossier, nous passerons à côté de ce qui relie ceux et celles qui se réunissent autour de ces musiques.

Il nous a donc semblé utile de parcourir deux « terrains » habituellement peu visités.

Le premier est celui par lequel tout commence et tout finit: le corps. Ce qui nous intéresse ici, c'est de comprendre les spécificités de la discipline techno et la façon dont les corps s'inscrivent dans quelques-uns de ses dispositifs. Pour le dire autrement, au lieu d'analyser le discours ou le "comportement » global des raveurs, il nous semble plus pertinent d'observer les modulations au sein de la foule. Nous avons notamment essayé de comprendre en quoi l'immersion dans la danse pouvait contribuer à une redéfinition des rôles en termes de genre et pour la communauté homosexuelle. L'examen montre que les stratégies sont variées.

Notre deuxième entrée aboutit dans l'autre espace de la techno, celui des (home) studios d'enregistrement et des machines de reproduction sonore: espace clos des chambres, des composants électroniques et des réseaux.

Il faut sortir ces techniques des boîtiers obscurs et s'intéresser à ce qu'elles font aux auditeurs et à la musique. On oublie trop souvent que le plaisir musical est bien instrumenté, inséparable des objets qui produisent la musique. Le corps dont nous parlions plus haut n'existe que dans une relation avec des machines musicales, il les joue autant qu'il est joué par elles. C'est cette relation, et son histoire, qui est au cœur des contributions qui suivent.

Justement, ce dossier s'ouvre par une sorte de boîte à outils. Nous avons demandé à Laure Delsaut, une des animatrices de la Médiathèque associative de Toulouse les Musicophages, un petit guide des ressources « on line» à l'intention des néophytes. On y trouvera une série de sites internet sur l'histoire du mouvement techno et des labels importants. Après cette introduction au domaine, le dossier est divisé en trois parties.

La première pourrait s'intituler «le corps de la techno».

Renaud Epstein et Astrid Fontaine se sont intéressés aux drogues, à leurs usages et eux régulations communautaires à l'œuvre dans les raves. Ils y montrent les stratégies des consommateurs pour contrôler et identifier les produits, leur imagination et leur usage des nouvelles technologies. Au-delà, ils s'interrogent sur l'usage des raves par les pouvoirs publics, qu'il s'agisse de réformer une politique toxicomanie obsolète ou d'envoyer des signes à l'opinion publique'.

\footnotetext{
1. À ce sujet, on peut rappeler que les décrets d'application de la loi sur la sécurité quotidienne, interdisant les free parties, ont été publiés à la veille du second tour de la dernière élection présidentielle par le gouvernement de Lionel Jospin. Gouvernement qui avait pourtant contribué à la légitimation de la scène techno à la fin des années 1990.
} 
On sait que la communauté gay a fortement contribué à l'installation du clubbing et de la house music en France, comme dans d'autre pays. Mouvements a donc rencontré Didier Lestrade, un des fondateurs d'Act Up, pour l'interroger sur les raisons de cette jonction et sur le contexte dans lequel elle s'était opérée.

De son côté, Vicky Amstrong fait le point sur l'insertion des femmes dans le mouvement techno en Angleterre. À partir des travaux d'auteures féministes, elle rend compte de la place des femmes sur les dance-floors et s'interroge sur les façons dont la technologie contribue (ou pas) au «partage des genres ». Sa contribution se conclue par une analyse critique du cyberféminisme.

La deuxième partie du dossier est consacrée aux technologies et à la reproduction sonore.

Jonathan Sterne remet en cause la différence traditionnellement opérée par les philosophes entre les médias et les instruments. II montre notamment combien les problématiques de la techno étaient déjà posées dès les débuts de la reproduction sonore. Son texte s'achève par une mini manifeste que I'on pourrait intituler: «pour en finir avec la fidélité ».

François Ribac souligne combien le rock, le hip hop et la techno ont fait du disque, et plus généralement des supports enregistrés, le matériau même de la passion musicale. II nous rappelle que quelques siècles avant les Beatles et Laurent Garnier, un autre support, le livre imprimé, avait déjà joué le rôle d'un instructeur. II montre enfin comment s'effectue le passage du privé au public.

Trevor Pinch retrace la carrière du synthétiseur moog, un instrument qui, un peu à la façon du piano au XIXe siècle, contribua de façon décisive à forger la sonorité des musiques des XXe et XXle siècles, techno incluse. Son texte s'achève par un (vibrant) hommage à son inventeur, Robert Moog, récemment disparu.

Le dossier se conclut par un entretien avec Simon Frith, une figure majeure des popular music studies. Celui-ci considère le mouvement techno dans I'histoire des musiques populaires. II montre combien les techniques numériques ont contribué à la fixation des répertoires et comment la figure du DJ s'est transformée, depuis les débuts de la house music. II établit un parallèle entre l'essor de cette musique et les métamorphoses récentes de l'industrie musicale. L'entretien se conclut par une réflexion sur les démarches respectives des cultural studies anglo-saxonnes et de la sociologie.

Last but not least, la rubrique livres accueille trois recensions d'ouvrages consacrés aux cultures électroniques: Un Maquis Techno de Sandy Queudrus, DJ Culture d'Ulf Poschardt et TAZ Zone Autonome Temporaire de Hakim Bey.

\section{Dossier coordonné par}

Renaud Epstein, Jean Paul Gaudillière, Irène Jami, Patricia Osganian et FrançoIs Ribac. 